A. Chauveau. Le travail musculaire et l'énergie qu'il représente. 1891 (Paris, Asselin et Houzeau), pp. 239-262.

Colın. Traité de physiologie comparée des animaux domestiques, t. 1, pp. 464-484. Heidenhann. Archiv für die Gesammte Physiologie, 1872, p. 309.

L. JUNG. a) Importance de la circulation à travers la mamelle áu repos ou en lactation chez la chèvre. C. R. Société de Biologie, t. 109, p. 737.

b) Particularités de la circulation mammaire chez la chèvre en lactation. T. 109 , p. 1052 .

L. Jung, R. Tagand et F. Chatanne. a) Innervation fréno-séerétoire de la muqueuse nasale. Soc. de Biologie, t. 106, p. 1315.

b) Sympathique cervical et sécrétion nasale. Soc. de Biologie, t. 107, p. 94.

LUDWIG. Zeitschrift für rat. Medizin, 1851, p. 259.

W. E. Petersen, L. S. Palmer et C. H. Eckles. La synthèse et la sécrétion de la matière grasse du lait. American Journal of Physiology, 1929, t. 90, pp. 573. 599.

Ch. Porcher. Traité de Physiologie normale et pathologique, publié sous la direction de H. Roger, t. 11, p. 294.

Ch. Porcher et L. JUNG. Intensité de la circulation mammaire chez la chèvre en lactation, O.R. I : 194. 1932, no 3, p. 234.

\title{
LA MÉTHODE A.I.V. POUR LA CONSERVATION DU FOURRAGE VERT
}

par

le Prof. Artturi I. VIRTANEN,

Conférence faite à Stockholm, pendant la Semaine agricole en Suède, le 15 mars 1932.

Le problème le plus important de l'économie rurale dans les pays où l'élevage du bétail laitier est développé, porte sur la question de l'azote. Dans les pays du Nord, la fertilité du sol est souvent limitée par l'insuffisance des substances azotées, et ainsi dans l'alimentation du bétail laitier et dans la production du lait, c'est encore le manque des aliments azotés, c'est-à-dire des albumines, qui est la cause des plus grandes difficultés. L'engrais azoté est le plus coûteux parmi les engrais, de même que les albumines forment la partie la plus coûteuse des substances nutritives. Une solution heureuse du problème de l'azote affecte done directement l'économie rurale. Le seul moyen de le résoudre dans les pays du Nord, c'est la culture des plantes légumineuses. Dans la conservation du fourrage vert, on rencontre de grandes difficultés, et de grosses pertes de la valeur nutritive sont inévitábles. Quand deux ou plusieurs fenaisons sont réalisées dans la même saison, - l'expérience a prouvé que cette façon de faire fournissait le fourrage le plus riche en albumines, - il n'existe de moyen de conserver chaque fenaison que par la dessic- 
cation, qui aboutit au foin. Il serait donc d'extrême importance de trouver une méthode qui permît de conserver le fourrage vert.

La conservation du fourrage vert par l'ensilage est une méthode ancienne. Cette conservation s'effectue, en effet, sans pertes notables, pourvu que le fourrage, préalablement haché, soit bien pressé, afin de faciliter le tassement de la matière et la sortie du suc des cellules. Il est parfois possible d'obtenir des résultats assez satisfaisants en accomplissant le travail soigneusement et en employant de bons réservoirs. La préparation de l'ensilage bien réussi dépend pourtant de plusieurs facteurs, et le risque de ne pas réussir est grand. Ordinairement, le silage, même dans les meilleures circonstances, ne peut être conservé sans pertes considérables en substances nutritives. Souvent ces pertes, d'après différents observateurs, varient notablement avec le procédé employé, le soin pendant le travail et l'espèce de plantes ensilées. En général, ces pertes sont égales à celles du foin séché, quoique des résultats tout à fait opposés soient aussi obtenus. Je vous renvoie aux expériences bien connues de WIEGNER, en Suisse, ainsi qu'à celles d'EDIN, en Suède.

Les pertes du fourrage ensilé proviennent pour une part de la respiration des cellules des plantes, mais elles sont dues principalement à l'action des différentes fermentations, et surtout à l'intervention des enzymes protéolytiques. Plus finement le fourrage est haché, plus fortement il est comprimé, d'autant plus faible est la respiration et d'autant supérieurs sont les résultats. Pourtant, on ne peut complètement éviter ni la fermentation, ni la protéolyse.

Les enzymes capables de décomposer les protéines se trouvent dans les cellules des plantes et dans les microorganismes, qui se développent rapidement en quelques jours, dans le fourrage. Au début du processus, le jus du fourrage étant encore neutre, les microorganismes produisant des enzymes protéolytiques et causant la décomposition rapide des protéines, se multiplient avee une grande vitesse. Parallèlement, se multiplient des bactéries qui attaquent les hydrates de earbone en produisant des acides; mais à mesure que l'acidité du fourrage augmente, l'action des bactéries décomposantes des protéines diminue. De la rapidité avec laquelle augmente l'acidité ainsi que de celle qui règle la décomposition des protéines, dépend le contenu du fourrage en protéines et en hydrates de carbone. Les pertes subies sont les plus grandes avec les fourrages riches en protéines, et souvent il arrive, dans ce cas, que le degré d'acidité ne monte jamais assez haut pour que la protéolyse soit interrompue. Cette décomposition des protéines est toujours accompagnée d'une odeur mauvaise, qu'on trouve souvent dans le silage ordinaire et qui peut même passer dans le lait. Les enzymes protéolytiques des plantes décomposent les protéines en acides 
aminés et en peptides ; l'ammoniaque peut être formée en petites quantités seulement en provenance de l'acide aspartique et de quelques amides. Certaines bactéries sont pourtant capables d'engendrer de l'ammoniaque, même en grandes quantités, aux dépens des produits de décomposition des protéines. Certes, les peptides et les acides aminés ont la même valeur nutritive que les vraies protéines, mais lorsque l'ensilage est fait dans des silos bon marché et non imperméables à l'eau, la formation de ces substances est la cause dè grandes pertes, parce qu'étant facilement solubles dans l'eau, elles peuvent s'échapper avec le jus du fourrage.

Même avec un ensilage dont le degré d'acidité monte suffisamment haut pour que la décomposition des protéines soit arrêtée, on observe de grandes pertes en protéines avant qu'une quantité suffisante d'acide soit formée. Les expériences effectuées par EDIN et SANDBERG, il y a plusieurs années, en sont un exemple excellent. Ces observateurs ont démontré que dans l'ensilage dont le $p H$ était 3,7 , il y avait une formation d'ammoniaque évaluée à $30 \%$ de l'azote total.

La formation des acides aux dépens des hydrates de carbone est due, au début, à l'activité des bactéries coli-aerogenes, ainsi qu'à celle des vraies bactéries lactiques. Celles-là produisent en grandes quantités de l'acide acétique et de l'acide formique, ainsi que de l'acide lactique ; celles-ci produisent presque uniquement de l'acide lactique. Les vraies bactéries lactiques dominent au-dessous du $p \mathbf{H} 4,5$; en décomposant les pentoses, elles produisent encore de l'acide acétique.

La formation de l'acide acétique et de l'acide lactique dans le silage est ainsi très facilement expliquée. De l'acide formique, on n'en trouve pas souvent en grandes quantités, parce que cet acide se décompose facilement, Le fourrage ensilé contient souvent de l'acide butyrique, qui est dû à l'activité du bacille butyrique. La formation de l'acide lactique n'est pas suivie d'une perte en valeur nutritive du fourrage. Mais par contre, on peut constater des pertes notables parallèlement à la formation de l'acide acétique.

$$
* * *
$$

Je vous ai présenté assez en détail les processus biologiques qui ont lieu dans le silage ordinaire, afin de rendre plus clairs les principes de la méthode A.I.V. La question la plus essentielle dans la conservation du fourrage est relative à la décomposition des substances azotées. Si la décomposition des protéines, d'une manière ou d'une autre, est entravée, la question de la conservation du fourrage sera ainsi résolue, pourvu, bien entendu, que la respiration des cellules des plantes soit en même temps arrêtée. 
En m'efforçant de trouver, en 1925, une méthode pour empêcher la décomposition des protéines du fourrage, je suis arrivé à ce résultat, que la manière la plus pratique et la plus économique serait, dès le début de la conservation, de faire augmenter la concentration des ions $\mathrm{H}$ dans le fourrage. Nos expériences au laboratoire de Valio ont démontré que ni les plantes ni les microorganismes anaérobies contiennent des enzymes protéolytiques eapables d'agir au-dessous du $p \mathrm{H} 4$. La conservation des protéines pourrait ainsi être admise à priori dans le cas où le degré de $p \mathrm{H}$ du fourrage, dès le début de la conservation, serait amené assez bas. Le développement du bacille butyrique sera de même entravé, puisque, selon nos expériences, il ne peut se multiplier dans une acidité aussi élevée que celle qui répond au $p \mathrm{H}$ ci-dessus.

Toutes les expériences entreprises au laboratoire de Valio dans le but d'élucider la question de la conservation, étaient basées sur lesdits points de vue. Mon collaborateur, Dr H. KaRStröM, m'a rendu de grands services en mettant ces expériences à exécution. D'abord, pour commencer, il s'agissait de connaître les quantités nécessaires de différents acides pour rendre le $p \mathrm{H}$ du fourrage $<4$. Si les quantités d'acide nécessaires se montraient très élevées, le procédé ne serait pas réalisable dans la pratique. Les résultats obtenus ont pourtant démontré que la conservation par un acide ajouté était en effet possible à réaliser. Nous avons vérifié qu'en employant des acides minéraux forts, 'comme l'acide chlorhydrique

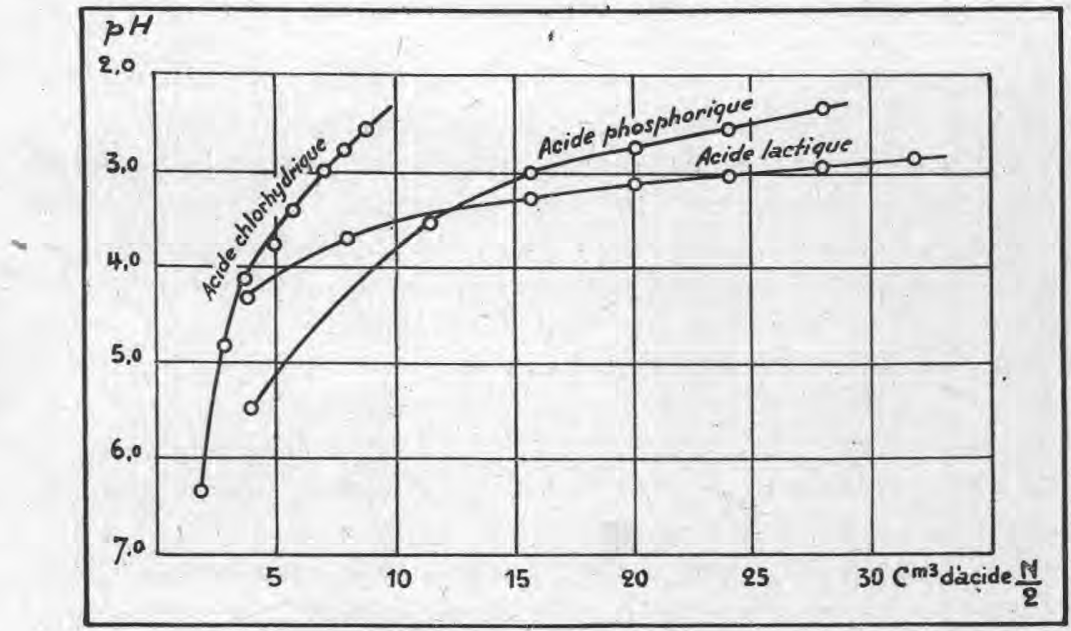

Fig. 1.

Expériences faites avec le seigle en herbe. - Action de l'acide chlorhydrique, de l'acide phosphorique et de l'acide lactique sur la concentration des ions hydrogène dans $100 \mathrm{gr}$. de fourrage vert. 
et l'acide sulfurique, on n'avait besoin d'en ajouter que de très petites quantités, afin d'obtenir dans le fourrage un $p \mathbf{H}<4$; qu'aucun trouble physiologique, au moins en ce qui concerne l'acide chlorhydrique, ne pouvait être lê résultat d'une semblable addition.

Les courbes des figures 1 et 2 indiquent clairement à quel point les différents acides augmentent l'acidité dans le fourrage.

Les expériences ont démontré que pour abaisser

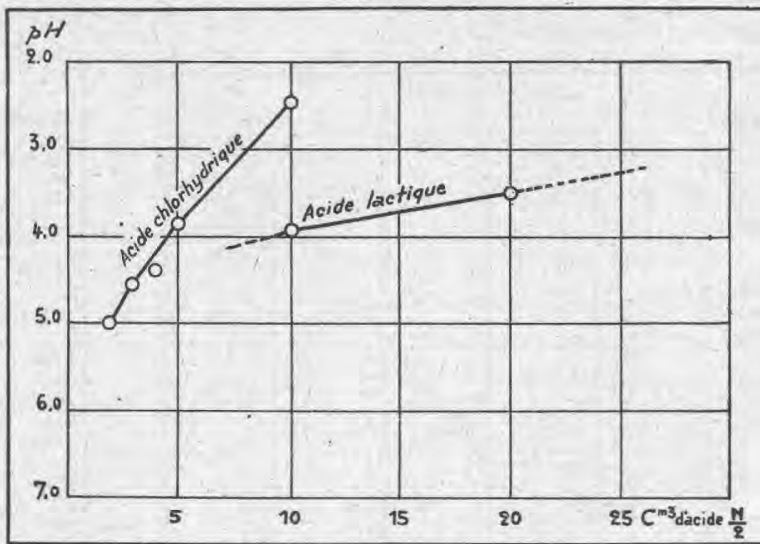

Fig. 2.

Expériences faites avec un mélange de trèfle et de phleum. - Action de l'acide ehlorhydrique et de l'acide lactique sur la concentration des ions hydrogène dans 100 gr. de fourrage vert.

.le $p H$ du fourrage, par exemple à 3,6 , l'emploi de l'acide lactique et de l'acide phosphorique exigeait une quantité de 4 à 7 fois plus grande pondéralement que celle de l'acide chlorhydrique.

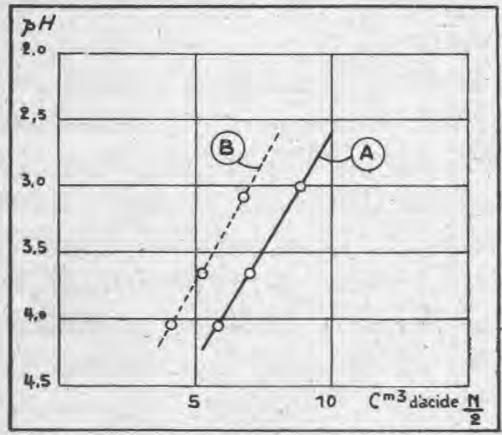

Fig. 3 .

Expériences faites avec le trèfle. Action de l'acide chlorhydrique sur la concentration en ions $\mathrm{H}$ dans une quantité de $100 \mathrm{gr}$. de fourrage vert :

A. Trèfle du sol dont le $p H$ a été 6,5 .

B. Trèfle du sol dont le $p H$ a été 5,5 .

Les courbes des figures précédentes montrent que des plantes différentes exigent des quantités différente d'acides pour que l'acidité ait le même $p H$. Sans exposer la question plus profondément, nous mentionnerons que les plantes légumineuses exigent plus d'acide que les graminées. Il est évident que c'est un fait d'importance dans la pratique.

La quantité d'acide n'est pas dépendante uniquement de l'espèce de la plante, mais elle l'est aussi de l'acidité du sol où lesdites plantes ont poussé, ainsi que de leur teneur en chaux. Ces faits sont montrés par la figure 3 .

Toutes ces expériences du 
laboratoire ont démontré que dans la conservation pratique du fourrage les aoides minéraux forts pouvaient être uniquement employés et que les différentes espèces de plantes exigeaient des quantités différentes d'acide.

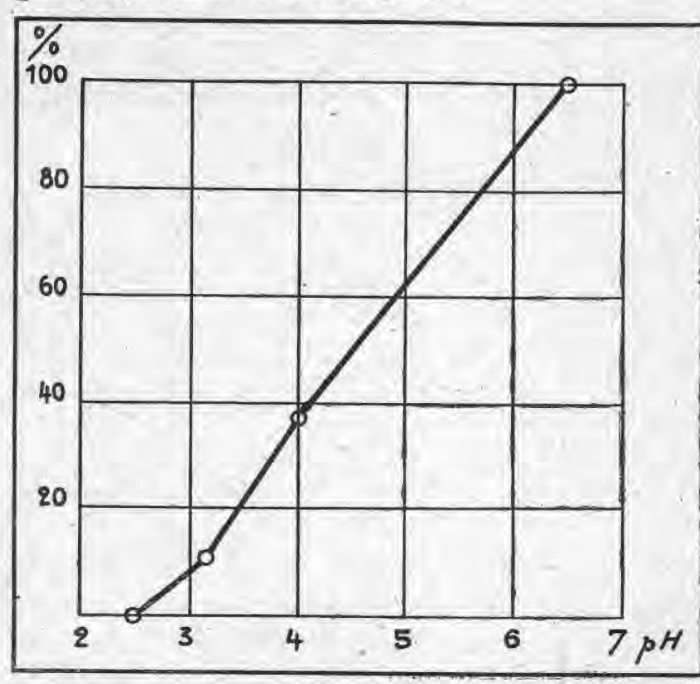

Fig. 4.

Expérience faite avec le poids vert. - Indique que

l'intensité de la respiration est dépendante de la concentration en ions $\mathbf{H}$.
Après avoir déterminé les quantités d'acide exigées pour obte. nir les différentes valeurs du $p \mathrm{H}$ du fourrage, nous nous sommes mis à l'étude de l'influence des différents degrés d'acidité sur la décomposition du fourrage. Par des expériences spéciales, on a constaté que la dépression de la respiration dans le fourrage était en relation avec l'augmentation du degré d'acidité et qu'elle était complètement interrompue au $p$ H 3. La courbe de la

figure 4 montre les résultats d'une expérience de respiration effectuée avee de jeunes plantes de pois verts.

Comme il sera constaté plus loin, la décomposition des protéines est entravée au-dessous du $p \mathrm{H} 4$ et, par conséquent, il était bien probable que la méthode que je préconisais pour la conservation du fourrage donnerait aussi dans la pratique des résultats excellents. On pouvait encore prévoir que non seulement les substances nutritives mais aussi les vitamines seraient bien conservées dans le fourrage artificiellement acidifié. Une réaction acide et un milieu anaérobie sont les conditions les plus favorables pour la conservation des vitamines.

Des expériences exécutées avec $25 \mathrm{~kg}$. de fourrage dans de plus grands réservoirs en ciment, nous ont donné des résultats qu'il eût été difficile d'acquérir au laboratoire. Tout d'abord, ees expériences ont démontré qu'il était très possible de faire un mélange homogène d'acide et de fourrage. D'autre part, il était possible de suivre les variations du $p H$ du fourrage pendant un temps plus long. Le tableau I montre les résultats d'une série d'expériences en employant ces réservoirs. Le fourrage était fait d'herbe jeune. 
TABLEAU I.

\begin{tabular}{|c|c|c|c|c|}
\hline \multirow{3}{*}{ Age du silage } & I & II & III & \multirow{3}{*}{ 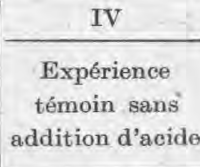 } \\
\hline & \multicolumn{3}{|c|}{$\begin{array}{l}\text { Addition d'acide chlorhydrique pour obtenir } \\
\text { un degré de } p \mathrm{H}\end{array}$} & \\
\hline & 3,2 & 3,7 & 4,2 & \\
\hline 25 jours & $p H=3.43$ & $p \mathrm{H}=3.68$ & - & $p \mathrm{H}=4.15$ \\
\hline 47 jours & $p \mathrm{H}=3.45$ & $p \mathrm{H}=3.52$ & $p \mathrm{H}=4.14$ & $p \mathrm{H}=4.09$ \\
\hline
\end{tabular}

Les expériences ont prouvé, par conséquent, que par l'addition d'acide, on pouvait en effet obtenir le degré de $p \mathrm{H}$ désiré et, au point de vue pratique, qu'il était resté invariable.

Les observations concernant la décomposition des protéines ont été faites de deux manières différentes.

Comme la protéolyse est toujours suivie de la formation de composés azotés solubles dans l'eau, la meilleure manière, à mon avis, de suivre les progrès de la déeomposition des protéines, était de déterminer la quantité d'azote soluble dans le silage. On trouve aussi dans le fourrage frais des composés azotés solubles, principalement des amides, qu'il faut prendre en considération pour apprécier les progrès de la protéolyse.

A notre laboratoire, l'azote soluble était dosé de la manière suivante :

25-30 gr. de fourrage, préalablement hâchés menu, étaient bien broyés avec de l'eau dans un mortier et ensuite quantitativement introduits dans un flacon de $100 \mathrm{~cm}^{3}$; après l'addition de $0 \mathrm{~cm}^{3} 5$ de formaline, ce flacon était empli à la marque 100 , bien agité et conservé durant une nuit à la glacière. La solution était ensuite filtrée sur linge à l'aide d'une pompe aspirante et soigneusement centrifugée. De eette solution transparente, l'azote était déterminé par la méthode de KJELDAHL.

Le tableau II nous donne quelques résultats expliquant la décomposition des protéines acquis au cours des essais exposés plus loin.

Ces chiffres montrent que les protéines sont bien conservées dans le fourrage de $p \mathrm{H} \mathrm{3,7}$, le taux en azote soluble et celui en azote ammoniacal, dans le fourrage de regain de trèfle, comme dans celui conservé durant 4 mois en $p$ H 3,7 , étant pratiquement les mêmes. Par contre, une protéolyse considérable a eu lieu dans le fourrage dont le $p \mathbf{H}$, par addition d'acide, a été 4,1. On peut en tirer la conclusion qu'il est d'extrême importance d'employer des quantités d'acide bien déterminées.

On peut conclure des expériences ci-dessus qu'il serait fâcheux de conserver le fourrage en employant des quantités d'acide au 
TABLEAU II.

DÉGOMPOSITION DES PROTÉINES DANS LE REGAIN DE TRÈFLE DURANT 4 MOIS.

\begin{tabular}{|c|c|c|c|c|c|}
\hline \multirow{2}{*}{ 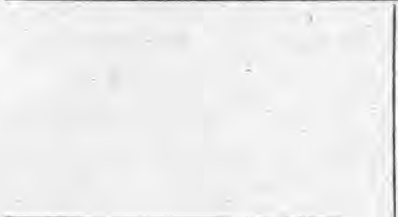 } & \multirow[b]{2}{*}{$\begin{array}{l}\text { Regain } \\
\text { frais }\end{array}$} & \multicolumn{3}{|c|}{ Addition de l'acide ehlorhydrique } & \multirow[b]{2}{*}{$\begin{array}{l}\text { Sans addi- } \\
\text { tion d'acide } \\
p \mathrm{H}=4,5 \\
\text { à la fin } \\
\end{array}$} \\
\hline & & $\begin{array}{c}p \mathrm{H} \text { au } \\
\text { début }=3.7 \\
p \mathrm{H} \text { à la fin } \\
=3,6\end{array}$ & $\begin{array}{c}p \text { H au } \\
\text { début }=4,1 \\
p H \text { à la fin } \\
=4,3\end{array}$ & $\begin{array}{c}p \mathrm{H} \text { au } \\
\text { début }=4,5 \\
p \mathrm{H} \text { à la fin } \\
=4,6\end{array}$ & \\
\hline 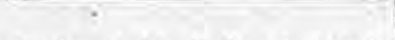 & & & - & & \\
\hline $\begin{array}{l}\text { Azote soluble de l'azote total : } \\
\qquad \% \ldots \ldots \ldots \ldots \ldots \ldots \ldots \ldots\end{array}$ & 26 & 28 & 44 & 60 & 65 \\
\hline $\begin{array}{l}\text { Azote ammoniacal de l'azote } \\
\text { total }: \% \ldots \ldots \ldots \ldots \ldots\end{array}$ & 1,5 & 2 & 12 & 21 & 22 \\
\hline
\end{tabular}

hasard. Ce fait a été la cause des insuccès des expériences exécutées en Allemagne, pendant ces dernières années, dans le but de conserver le fourrage par l'addition d'acide chlorhydrique. ScHweIzer fait ressortir qu'en employant des quantités minimes d'acide, le fourrage s'est altéré, et par contre qu'en employant des quantités plus grandes, le bétail, ou bien se refuse à consommer ce fourrage, ou bien, s'il le consomme, tombe malade au bout de quelques jours et commence à dépérir. SchweIzer lui-même écrit : "Nous nous sommes une fois de plus assuré du fait, que le bétail ne peut être alimenté en quantités suffisantes de fourrage acidifié, à cause de la grande quantité d'acide de ce fourrage. »

FingERLing a cherché à conserver le fourrage vert en le plongeant dans une solution d'acide chlorhydrique. D'après sa dernière demande de brevet, le $p H$ dans cette solution devait être maintenu à 2 , pendant toute la période de la conservation. Sans compter qu'une telle méthode ne pourrait être réalisable dans la pratique, la quantité d'acide qui sera nécessaire pour maintenir l'acidité au $p$ H 2 (fig. 1 et 2), est au point de vue physiologique nuisible au bétail. Les expériences de FINGERLING n'ont pas donné de résultats positifs non plus, selon le livre : "Die Silofutterbereitung nach dem Kaltgärverfahren ", publié en 1930 par les auteurs KIRScH et HILDEBRANDT, Lesdits auteurs écrivent entre autre : "Il est désespérant de proposer l'emploi d'acide chlorhydrique pour la conservation du fourrage. " Pourtant, à présent, les principes de ma méthode étant connus, le fait paraît assurément bien simple (1).

Comme l'intensité de la respiration au $p \mathrm{H} 3,6$ est réduite de

(1) Les docteurs HILdEBRANDT et Krrsch de Königsberg sont venus en Finlande au printemps 1931 dans le but de faire connaissance avec la méthode A.I.V. Au retour de ce voyage ils prétendent avoir trouvé eux-mêmes une nouvelle méthode (nommé la méthode « Defu »). Cette méthode ne semble qu'une imitation de la méthode A.I.V. 
$80 \%$, et la décomposition des protéines étant, en même temps, complètement entravée, lesdites opérations ne peuvent être suivies de pertes notables en substances nutritives du fourrage. Les changements de la teneur en hydrates de carbone dans le fourrage sont aussi très minimes avec ladite concentration en ions $H$. Certains streptocoques et bâtonnets, étant capables de faire fermenter aussi des pentoses, se trouvent encore dans un fourrage à $p \mathrm{H}$ d'environ 3,6 , mais les fermentations causées par lesdites bactéries qui vivent à un tel degré d'acidité, sont pourtant très faibles. Dans le fourrage A. I. V. l'acide lactique est régulièrement trouvé en petites quantités, ce fait étant dû soit à la fermentation microbienne soit à l'activité des cellules des plantes. Cette formation de l'acide lactique n'occasionne pourtant pas de pertes nutritives, la valeur nutritive du sucre et eelle de l'acide lactique, aux concentrations en question, étant à peu près les mêmes. D'ailleurs, une formation d'acide lactique aux dépens des pentoses, procure un gain en substances nutritives digestibles.

En ce qui eoncerne les acides volatils, une minime quantité d'acide acétique est formée, en provenance soit des pentoses soit des substances à pectine. Il faut spécialement observer qu'en déterminant la quantité d'acide acétique du fourrage acidifié, la méthode générale de distillation à la vapeur d'eau ne peut, en ce cas, être employée, ear lors de l'emploi dudit procédé, l'acide acétique se détache de certaines substances de plantes, probablement des pectines. Pour cette raison, nous avons, ces derniers temps, extrait à l'éther du fourrage acidifié les acides gras volatils et l'acide acétique. Les acides volatils étaient ensuite distillés à la vapeur d'eau et l'acide lactique isolé du résidu comme lactate de zinc. Le tableau III donne les résultats de trois analyses faites de cette manière.

TABLEAU III.

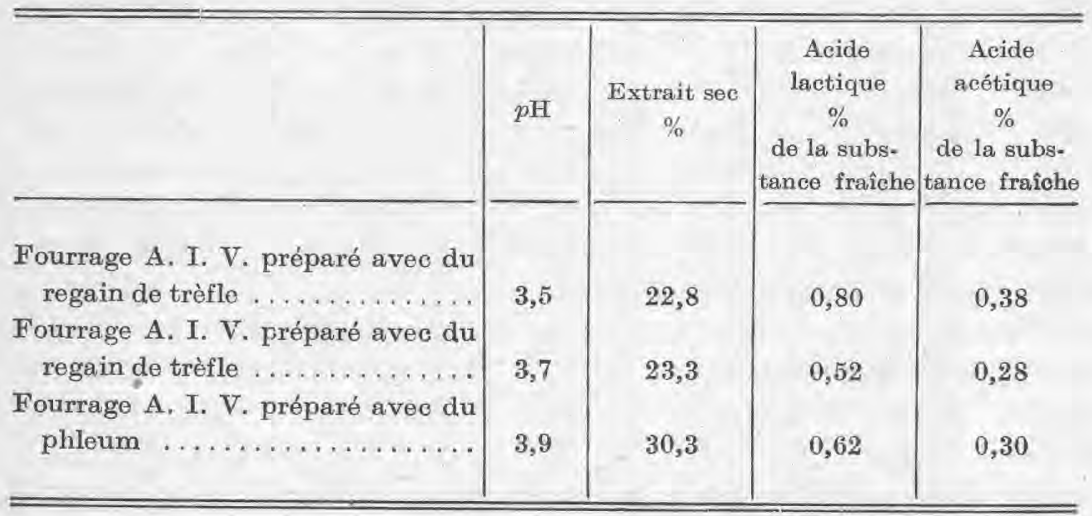


L'acide butyrique n'a jamais été trouvé dans un fourrage dont le $p H$, tout au début de la conservation, est ramené au-dessous de 4.

Pour affirmer ultérieurement l'insignifiance des procédés de fermentation dans le fourrage A. I. V. acidifié au-dessous du $p H$ 4, il faut observer que ce fourrage contient toujours des sucres réducteurs, lesquels ne sont jamais trouvés dans l'ensilage ordinaire. Les chiffres du tableau IV indiquent le taux en sucres réducteurs des différents échantillons du fourrage A. I. V.

TABLEAU IV.

SUCRE RÉDUGTEUR : \% DE L'EXTRAIT SEC.

\begin{tabular}{c|c}
\hline $\begin{array}{c}\text { Fourrage A.I.V. préparé avec du trèłle } \\
\text { et du phleum. I fenaison }\end{array}$ & $\begin{array}{c}\text { Fourrage A.I.V. préparé avec du regain } \\
\text { de trèłle }\end{array}$ \\
\cline { 2 - 2 } 1,4 & 6,5 \\
2,5 & 1,4 \\
1,9 & 2,0 \\
& 3,5 \\
\hline \hline
\end{tabular}

Quelles sont les sources d'acidité dans le fourrage acidifié par l'acide chlorhydrique ? Voilà un fait qui n'est pas aussi facile à déterminer qu'on pourrait se l'imaginer de prime abord. Il a été montré que bien que le fourrage ait été acidifié par l'acide sulfurique ou par l'acide chlorhydrique à un $p \mathrm{H} 3,6$, la réaction des cendres du fourrage est restée alcaline. Il est par suite évident que les acides minéraux du fourrage existent non pas à l'état libre, mais sous forme de sels. Il est évident que les acides minéraux forts réagissent sur les éléments alcalins du fourrage, en libérant des acides organiques plus faibles, comme l'acide oxalique, l'acide malique et l'acide citrique, qui ainsi augmentent le degré d'acidité du fourrage. Nous avons déjà mentionné cí-dessus que, plus tard, de petites quantités d'acide lactique et d'acide acétique sont également formées.

Nous mentionnerons encore que les quantités d'acide lactique et d'acide acétique, se trouvant dans le fourrage acidifié par l'acide chlorhydrique, ne peuvent avoir aucun effet notable sur l'acidité du fourrage conservé. Selon les courbes des figures 1 et 2 , l'augmentation de la concentration en ions $\mathrm{H}$, occasionnée par l'acide lac tique, très faible, est extrêmement faible entre les $p \mathrm{H} 3$ et 4 . L'influence de l'acide acétique, qui est encore plus faible que celle de l'acide lactique, est absolument sans importance. Par cette raison, les petites quantités d'acide lactique et d'acide acétique formées par la fermentation ne peuvent suffire pour conserver le fourrage.

Le fait que les acides minéraux forts se trouvent uniquement 
sous forme de sels, est au point de vue physiologique d'une très grande importance et donne la possibilité d'alimenter le bétail uniquement avec du fourrage conservé par les acides, sans dérangement quelconque.

(A suivre).

\title{
SUR LA THÉORIE DE LA POLYMÉRISATION DE LA CASÉINE.
}

\author{
par MaURICE BEAU \\ Ingénieur agronome.
}

Nous avons exposé dans un précédent article (1) une nouvelle théorie permettant de rendre compte d'une façon simple des phénomènes qui se passent dans la coagulation de la caséine par la présure, et dans la transformation de la caséine en paracaséine.

Cette théorie consiste en résumé à dire :

$1^{0}$ que, dans la easéine telle qu'elle sa trouve dans le lait, il y a liaison entre un certain nombre de groupes $\mathrm{COOH}$ d'une molécule et un nombre égal de groupes $\mathrm{NH}^{2}$ d'une autre molécule, d'après un schéma analogue au suivant :

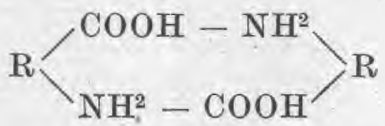

(Ce sont probablement ces liaisons d'ordre chimique qui, se répétant sur un nombre considérable de molécules, arrivent à constituer les agrégats physiques qui, sous le nom de micelles de caséine, sont visibles à l'ultra-microscope.)

$2^{\circ}$ que l'action de la présure consiste d'abord à seinder ces liaisons, de manière à rendre de nouveau libres les groupements $\mathrm{COOH}$ et $\mathrm{NH}^{2}$, qui peuvent alors se combiner avec les radicaux $\mathrm{CaO}$ et $\mathrm{P}^{2} \mathrm{O}^{5}$ présents dans le liquide, pour donner le phosphoeaséinate de chaux en agrégats physiques de plus en plus volumineux et polymérisés, qui constituent le bloc de caillé, et qui donnent après séchage la caséine dite à la présure.

Si cette théorie est exacte, il en résulte que le premier effet de la présure doit être d'augmenter simultanément l'acidité et l'alcalinité de la caséine, par suite de la mise en liberté, à la fois, de valences $\mathrm{COOH}$ et $\mathrm{NH}^{2}$. Mais il va de soi qu'il n'est pas facile de mettre ce résultat en évidence; en effet, si on se borne à titrer à la manière ordinaire l'acidité moléculaire totale ou potentielle de la easéine, surtout en opérant avec des acides ou des alcalis forts, on ne trouve aucune différence, la caséine et la paracaséine se comportant dans ce cas comme des corps entièrement dépolymérisés.

(1) Le Lait, juillet-août 1932. 